\title{
A Study on Translation Textbooks for Continuing Education
}

\author{
Yanqun Zou \\ Beijing International Studies University \\ Beijing, China
}

\begin{abstract}
Translation textbooks reflect the core concepts and principles of the subject, and play a significant role in developing students' translation competence. However, the quality of textbooks varies, which brings negative impact to translation education. The research team on translation competence development conducted a survey on translation textbooks and learning to explore guidelines for translation textbook for continuing education based on the statistics of the survey.
\end{abstract}

\section{Keywords-translation competence; ALERT mode; translation textbook}

\section{INTRODUCTION}

Translation textbooks reflect the core concepts and principles of the subject, and play a significant role in developing students' translation competence (TC). Textbooks are not only supposed to be used for class and review, but to mirror the essential knowledge and concepts of translation in an organized and brief way. (Zhang Meifang, 2001:154-155)

Translation study comes into the blooming period since 1980s in China, and translation textbooks proliferate fast. Then scholars and experts have realized the necessity and urgency of the study on textbooks. Professor Zhang Meifang's study on E-C translation textbooks in China discusses the categories, the development and tendency, the comments from users, and the building of textbooks comprehensively since 1980s. Professor Liu Jichun puts forward the compiling principles of translation textbooks: principle of advanced and updated translation knowledge and principle of descriptivism. Zhang Yu presents four principles about translation textbooks: combination of theory and practice, keeping up with the times, introduction of both Chinese and Western translation views, and marketorientation. Professor Yang Zijian holds that compilers are supposed to think about the system of knowledge, system of theory, and system of training to compose textbooks with clear logic. Wang Shuhuai puts forward systematic principle and interlinking principle. Wang Jianguo and Tao Youlan make use of functional theory and skopos theory to interpret the guidelines of compiling translation textbooks. A great number of articles discuss the role of theory and practice, and the proportion of theory in textbooks. (He Ruiqing\& Huang Chuanying, 2013)
According to Dr. Tao Youlan's survey, translation textbooks are over 1000 in the market in China. However, the quality of textbooks varies, the compiling frames are always outmoded, the compiling notion, content, and structure of textbooks are repetitive and lack of innovation, which bring negative impact to translation education. (Tao Youlan, 2008) Firstly, the frame of textbooks lacks of macroscopic view. The design of textbook frame always follows the outmoded structure: basic theories and translation techniques; or basic knowledge, translation of words, sentences, and passages; in addition, the chapters lack of logical relation and interaction. Besides, theories and practice are not harmoniously related. Secondly, a majority of textbooks are confined to the microcosmic level-the transfer of languages, and rarely cover the relevant external factors, and fail to put translation under the social context and other related disciplines. Thirdly, most traditional textbooks are prescriptivism-oriented. In teaching translation techniques, the textbooks only offer the reference translation text without interpretation and translation process; in the introduction of translation criteria, the textbooks merely present the principle of sincerity, not involving other translation concepts. Hence what readers obtain is no more than the translated text without the ways to reach the ideal translated text, which is not sufficient to improve readers' translation competence effectively and efficiently.

Some scholars bring forward that translation textbooks are supposed to be developed according to students' levels, while most textbooks are designed for regular undergraduate students and postgraduate students, and textbooks for undergraduates in continuing education are very few. In addition, students in continuing education face the sharp contradiction between limited class hours and numerous, complicated content; and the contradiction between practice and diverse, sophisticated theories. Therefore the study on translation textbooks for students in continuing education becomes an urgent issue.

\section{SURVEY ON TRANSLATION TEXTBOOKS AND LEARNING}

The research team on translation competence development conducted a survey on translation textbooks and learning in the School of Continuing Education of Beijing International Studies University in 2017, and collects 136 valid questionnaires, concerning the basic information of 
respondents, the motive of translation learning, the necessity of translation class, time on translation learning, difficulties in translation learning, understanding of translation competence, preferred learning texts and materials and so on. We will explore the preferred texts and guidelines of translation textbook for continuing education based on the statistics of the survey.

\section{A. Basic Information and Characteristics of Students in School of Continuing Education}

1) Age: 136 respondents range from 18 to 40+ years old, while students ranging from 23-31 account for 81\%(110 respondents). And 89\% (121 respondents ) are female. Teachers are suggested to concern the complicated factors on the physiological \& psychological features in textbook design. The ability to learn declarative knowledge might not be as strong as regular undergraduate, while they have a deeper understanding on the importance of reflection and regulation because of their social and working experience, thus teachers are supposed to elaborate their advantages, and guide them to improve the regulating ability so as to promote their translation competence.

2) Working field: The respondents' working fields concern tourism, trade, finance, law, education, government and so on. The respondents working in trade account for over $50 \%$, and respondents working in education account for almost $20 \%$, thus teachers are supposed to correlate the students' needs in teaching materials and class design, laying importance in the characteristics of translating material in trade and education.

3) Motive of learning translation: With regard to the motive to learn translation, about $40 \%$ respondents learn translation because they are required to translate materials in work, and about $50 \%$ respondents hope to learn more about the world in English. While concerning the working needs of students, teachers are required to cover different fields of materials to broaden students' vision.

4) The necessity of translation class and importance of translation competence: As for the necessity of translation class, $98.5 \%$ respondents think that it is necessary to have translation class, which means that respondents have clear understanding on the necessity of translation class. $95.6 \%$ respondents have realized the importance of TC, and hope to improve TC.

5) Significance of TB: With regard to the importance of TB, 86.4\% (108 respondents) consider TB very important, $12 \%$ (15) consider TB important, and $1.6 \%$ (2) consider TB not important, which shows that most respondents have realized the importance of TB.

6) Time on learning translation: As for the time spent on learning translation each week, $22.1 \%$ respondents say they do not learn on translation at all, $51.4 \%$ respondents spend less than one hour on translation, $11 \%$ respondents spend two hours on translation, which indicate that most respondents spent less than one hour on translation each week, which is far from being enough to make progress in translation.

\section{B. Understanding about the Constitution of Translation Competence}

1) Constitution of translation competence: As for the constitution of translation competence, linguistic competence, pragmatic competence, intercultural competence, theoretical competence and textual competence are the first five choices, and the followings are coordinative competence, information competence, strategic competence, research competence and professional ethics. Few respondents chose subject knowledge, profession standard and management, conscience and critical thinking.

2) Competence that translation class needs to set priorities on: As for the most important competence that translation class needs to develop, the first five choices are bilingual competence, pragmatic competence, intercultural competence, theoretical competence and textual competence, and the followings are coordinative competence, information competence, strategic competence, research competence and critical thinking. The statistics of this question echo the statistics of the previous questionrespondents tend to lay more emphasis on linguistic competence, but pay less attention to information competence, subject knowledge, and the core competence in translation-strategic competence.

3) Extracurricular reading and translation competence: $86 \%$ respondents say that extracurricular reading are significant to improve their translation competence, to which teachers are highly suggested to offer positive encouragement and guidance to show the meaning of extracurricular reading to the promotion of field knowledge, understanding and writing to enforce students' initiative of reading.

4) The relation between writing and translation: $86 \%$ respondents say that the improvement in English writing is helpful to the promotion of translation competence, which suggests that most respondents have realized the positive correlation between English writing and translation.

5) The correlation between parallel text and translation competence: $80 \%$ respondents show that the reference to parallel texts offers effective assistance to translation. Teachers are required to introduce parallel texts and corpora to show the effectiveness of parallel texts in promoting translation quality, efficiency, and stimulate students to construct relevant corpora to improve their sense of satisfaction in learning, and promote their strategic competence, technical competence, and translation competence effectively.

6) Translation and communication: Two third respondents indicate that learning translation is beneficial to promote their communicative skills, and vice versa, which demonstrates that most respondents have recognized that 
current translation requires more than professional translation knowledge and skills.

7) Translation and cooperation: $88.3 \%$ respondents say that learning translation helps improving their cooperative ability, and vice versa, thus we could infer that most respondents have realized that translation is no longer a single-handed work, which requires team work and cooperation. Half respondents show that they prefer to group learning compared to studying alone in translation learning, which indicates that these respondents have certain understanding about the characteristics of translation in current circumstances, which does not only require ability in word processing.

8) Other relevant abilities: $86 \%$ respondents reveal that effective translation requires the ability to understand others.

$81.4 \%$ respondents indicate that translators are supposed to be conscientious besides being competent in professional skills.

\section{Preferred Learning Texts}

1) Difficulties in translation: About the difficulties in translation, the most frequent choices are sentence structure, cultural differences, pragmatic problems, grammatical problems, and vocabulary; and the followings are cohesion, textual structure, comprehension of text and reader difference, which shows that the respondents pay more attention to the translation of sentences - sentence structure, grammar, vocabulary, and the mention of cultural difference is a delectable change; however, they lay less emphasis on textual structure and cohesion, and do not have clear understanding about the significance of reader difference and the accuracy of comprehension. Thus, we need to guide students lay more emphasis on the structure and cohesion of text on the basis of sentence translation according to the initiator, goal, target reader of translation; furthermore, the communicative translation theory is supposed to be introduced to update students understanding of translation essence to understand translation in perspective of intercultural communication.

2) Texts of interest and need: The most frequent choices of the texts of interest are touristic text, news and reports on the state of the country, fiction, scientific and technical texts, and the followings include economic and financial texts, legal texts, ancient classics. As for the texts that respondents need to deal with, economic and financial texts, touristic texts, news and reports and legal texts are the most frequent choices, and the followings are ancient classics and fiction. Consequently we are supposed to give consideration to the students' need and interest in choosing learning materials, stimulate students' interest by touristic text and news, introduce diverse tools such as corpora to improve translation efficiency and effect.

3) Expectation on textbook: For the ideal textbook, the most frequent choices are introduction by texts, the introduction of translation skills and strategies, the interpretation of sentences, the interpretation of text and passage, and the introduction of translation theories, which show that respondents have had certain understanding of text types, hoping to learn translation skills and strategies, and paying more attention to the translation of sentence. Therefore, we are encouraged to enforce the characteristics of different text types, introduce translation competence besides translation strategies, and integrate strategic competence, instrumental competence and subject knowledge in the process of translation to guide students to concern different factors of translation in terms of text and passage from communicative perspective on the basis of sentence.

\section{CONCLUSION}

The study on translation textbooks is an essential part of translation education, which is required to be studentoriented to establish the right view on translation, to enhance translation competence, especial the metacongnitive and strategic competence to solve various and dynamic problems in the current context. We are encouraged to follow the following principles as for the selection of texts.

Firstly, applied materials are the priorities. According to the survey, the current requirements the respondents confront have turned from material of literature and social sciences to applied materials. Therefore the major part of translation textbooks for continuing education are required to focus on applied translation, choosing practical, typical, and interesting materials to stimulate students' motive and learn to apply in their work. We need to think of the feasibility of the materials, choosing the appropriate materials in accordance with students' cognitive level, from simple texts to sophisticated and complicated ones; take both students' needs in work and leisure and interest into consideration.

Secondly, the frame of textbooks is designed on subjects. In the light of the survey, the most frequent choice is introduction by subjects and themes for ideal textbooks. Therefore, updated textbooks are encouraged to break the original grammatical frame to compile the materials in accordance to different subjects and themes. The updated textbooks will introduce touristic texts, economic and financial texts, news and reports on the state of the country, scientific and technical texts, legal texts, ancient classics and fiction. Thus, teachers are supposed to give consideration to the students' need and interest in choosing learning materials, stimulate students' interest by touristic text and news, introduce diverse tools such as corpora to improve translation efficiency and effect; and integrate strategic competence, instrumental competence and subject knowledge in the process of translation to guide students to concern different factors of translation in terms of text and passage from communicative perspective on the basis of sentence.

Thirdly, the requirements of compilers. Compilers are to be familiar with translation theories, relevant disciplines, the advantages and disadvantages of current translation textbooks; be familiar with the teaching principles, 
understand students' expectations, thinking process and tendency in order to interpret the sophisticated translation theories briefly to stimulate their interest to study and practice them in their own works.

The compiling of textbooks is encouraged to follow the general guideline: with the guide of theories, translation textbooks are to build students' view on translation and develop their translation competence based on the comparison between Chinese and English and the introduction of translation techniques on the ground of social needs. The compiling of translation textbooks are supposed to refer to the following principles.

Firstly, the development of understanding on translation and translation competence is supposed to be integrated into each chapter through the textbook. The understanding of translation determines the choices translators make in the process of translation, and exerts influence on the quality of translation. The lucid and sophisticated understanding on the essence of translation leads students on the way to develop their translation competence and promote the effectiveness and efficiency of translation, even though they are not able to produce excellent translated version right now. On the contrary, with ambiguous, one-sided understanding on translation, students would not be able to reach the expected goals even with a great quantity of practice. Besides, the development of translation competence is a long and gradual process on the ground of the development of linguistic knowledge, subject knowledge, instrumental knowledge, and especially the strategic competence. The lucid and sophisticated understanding of translation plays a more significant role than practice in the long run. (Liu Jichun, 2017)

We are supposed to turn from microcosmic view to macrocosmic view, to introduce skopos theory, which emphasizes the dynamic, situational and contextual features of translation, to broaden and deepen students' understanding on translation in the current context. In addition to the introduction of linguistics, cultures, sociology, anthropology and other relevant disciplines are suggested to be added. Besides the microcosmic linguistic problems, the related external factors are required to be analyzed thoroughly. Applied translation is always to realize a practical, concrete purpose, and the purpose and function are the ultimate target. Hence skopos theory provides feasible guidelines to applied translation in terms of the selection of information from the original text, the choice of translation strategies, the style of the text and so on.

Secondly, translation textbooks are supposed to be descriptivism-oriented. Scholars have reached the agreement that theories are the indispensible part of translation textbooks, and the point is how to integrate theories into practice harmoniously. The skopos theory, functional theory, and text type theory are the effective theories to guide applied translation, which are to be elaborated thoroughly in the textbooks for continuing education, while the other relevant theories are encouraged to be introduced accordingly for students to explore. In terms of translation strategies, various strategies are supposed to be covered, including abridged translation, edition-translation, transwriting, rewriting, interpretation and so on. Students are encouraged to rewrite according to the purpose of translation, readers' expectations, the receiving medium, place and time. In interpreting the translation examples, we should not only present the reference version, but to show the process of translation.

Thirdly, based on ALERT mode, introduce reflective journal, project-driven teaching and workshop, to integrate translation brief through the process of teaching to develop students translation competence comprehensively, especially the metacognitive competence. Students are required to translate each text with the employment of ALERT mode. The clarification of constitution of translation competence would awaken students' consciousness on other competence - subject competence, instrumental competence, intercultural competence, cooperative competence, metacognitive competence, besides linguistic competence. Hence the introduction of PACTE's view of translation competence is the prerequisite. Furthermore, most translation textbooks merely offer the reference text and focus on the result of translation, fail to analyze the process of translation. The introduction of ALERT mode would remedy the disadvantage. We are to highlight the significance of metacognition before concrete translation activities; integrate model ALERT in class translation activities, analyzing, locating problems, exploring strategies, run the strategies, and tracking and monitoring in the process of translation; reinforce and reflect on the performance with translation journal after class. ALERT mode helps students realize that the completion of each translation task is not the ultimate purpose, to develop the translation competence by monitoring, regulating, and reflecting on the performance is the goal; what they are supposed to learn is not only declarative knowledge concerning languages, cultures, instruments, professional knowledge on translation, but also procedural and conditional knowledge about maximizing and optimizing the resources accessible to enhance their translation competence. Metacognition equips students to satisfy the needs of the age, to meet the requirement of translation for the current context; metacognition is the necessary power for students to become qualified and competent translators.

The adoption of task-based and project-based teaching methods would offer students opportunities to encounter the possible difficulties and challenges. We could even offer more than one translation brief, so that students may produce different versions accordingly to develop the understanding of the essence of translation, especially applied translation. Peer-review offers another opportunity for students to reflect on their translation process. 


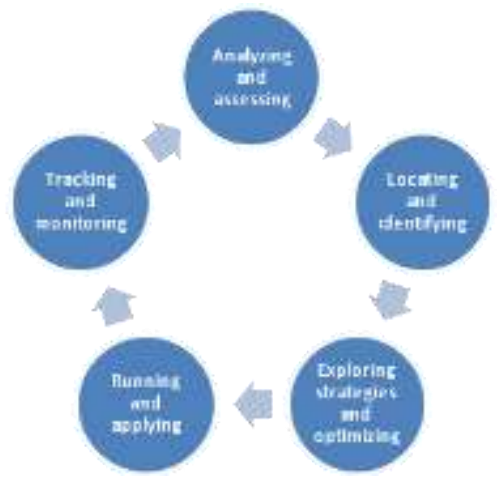

Fig. 1. The process of translation.

Fourthly, the update of textbooks with the assistance of MOOC, corpora and website. With the development of translation studies and translation market, the essential concepts and selected materials may need to update accordingly, therefore, the employment of website and relevant tools are the tendency. We are supposed to combine computer-assisted teaching and translation organically, so that students could make full use of their fragmented time to learn translation by MOOC, and compilers could update selected materials on time, textbook users could choose materials from corpus to realize the electronization, diversification, and multi-dimensionality of translation textbooks, to use textbooks creatively.

With limited class hours, students in school of continuing education are to give full play of their advantage in work, to develop their translation competence according to the needs in work, to integrate study with work harmonious, so that there would be virtuous circulation, and study and work would complement each other.

\section{REFERENCES}

[1] Zhang Meifang, English Chinese Translation Textbooks in China[M], Shanghai Foreign Language Education Press, Shanghai, China, 2001,pp164.

[2] Zhang Meifang, "Study on translation textbooks to promote translation education" [J], Shanghai Journal of Translators, 2015(2), pp 54-57.

[3] He Ruiqing\& Huang Chuanying, "The cohesion of translation textbooks of undergraduates and MTI'[J], Chinese Science \& Technology Translators Journal, 2013(4) , pp33-36.

[4] Liu Jichun, "Some observations on the establishment of a new hierarchical system for translation textbooks in China" [J], Chinese Translators Journal, 2001(4),pp49-53.

[5] Zhang Yu," Translation teaching tendency in China in the era of globalization" [J], Foreign Language World, 2003(1), pp13-16.

[6] Yang Zijian, "Some issues on translation education" [J], Shanghai Journal of Translators, 2006(3),pp36-40.

[7] Wang Shuhuai, "On the principles of writing Chinese-English translation coursebooks" [J], Foreign Language Learning Theory and Practice, 2011 (2), pp85-91.

[8] Wang Jianguo, "On translation textbook compilation in China from the perspective of the functionalist approaches to translation" [J], Language and Translation, 2004(2), pp58-60.

[9] Tao Youlan, "On the E-C\& C-E translation textbooks in the perspective of Skopos theory" [J], Foreign Language World, 2006(5), pp 33-40.
[10] Tao Youlan, "Exploration on a new type of C-E translation textbooks for the newly established translation Specialty" $[\mathrm{J}]$, Foreign Language World, 2008(2), pp10-19.

[11] Tao Youlan, Translation Textbooks in China: A Theoretical Reconsideration of Their Making[M], Shanghai Fudan University Press, Shanghai, China, 2008.

[12] Tao Youlan, "An integrated approach toward teaching MTI students translation with focus on improving translator competence" [J], Foreign Language Learning Theory and Practice, 2015(4), pp87-91.

[13] Liu Jichun, "The rationale of the making of A glimpse of translation: theory and practice" [J], Shanghai Journal of Translators, 2017(6), pp67-73.

[14] Zou Yanqun, "The concept and instruction of metacognition in translation competence development" $[\mathrm{J}]$, International Forum of Teaching and Studies 2015(2), pp69-78. 